\title{
Dual-wavelength thulium-doped fiber laser assisted by non-adiabatic tapered fiber
}

\begin{abstract}
A dual-wavelength ring cavity thulium-doped fiber laser (TDFL) assisted by tapered optical fiber was experimentally demonstrated. The tapered optical fiber acted as a comb filter to provide seed signal and limit oscillating modes, which minimized gain competition and ensured stable operation. Lasing thresholds were reached at low pump powers for both single wavelength and dual-wavelength lasing. The single wavelength laser was obtained at $60 \mathrm{~mW}$ while dual-wavelength output was obtained at $100 \mathrm{~mW}$. The peaks of the dual-wavelength laser occurred at $1958 \mathrm{~nm}$ and $1980 \mathrm{~nm}$ with optical signal-to-noise ratio around $67 \mathrm{~dB}$. The maximum power difference between the two peaks was less than $1.2 \mathrm{~dB}$ and power fluctuations over a 45 -minute period did not exceed $3 \mathrm{~dB}$. The proposed work offers stable dual-wavelength output with excellent performance in $2-\mu \mathrm{m}$ band using a simple laser configuration. The success of this work further facilitates the deployment of $2-\mu \mathrm{m}$ fiber lasers for real world applications.
\end{abstract}

Keyword: Fiber lasers; Thulium-doped fiber; Multiwavelength; Optical filters 
